\title{
COMPETITIVIDADE DA SOJICULTURA EM MOÇAMBIQUE: UMA ANÁLISE BASEADA NA EXPERIÊNCIA DE PRODUTORES LOCAIS
}

Ricardina Janeque ${ }^{1}$ Nilson Costa ${ }^{2}$

Resumo: A sojicultura, em nível global, está entre as atividades econômicas que, nas últimas décadas, apresentaram crescimentos mais expressivos, sobretudo no campo dos agronegócios. Por isso, objetivou-se analisar a competitividade da sojicultura moçambicana a partir de técnicas de estatística multivariada, baseandose na teoria da competitividade sistêmica. A pesquisa utilizou dados primários, obtidos no período de janeiro à março de 2018, em Moçambique. A amostra foi de 120 sojicultores, em que a técnica de amostragem foi não probabilística e a escolha dos produtores foi a partir da técnica "bola de neve". Teve-se como referência técnicas de estatísticas descritiva e de estatística multivariada (Análise Fatorial Exploratória e Análise de Conglomerados) para compilação e tratamento dos dados. A análise fatorial possibilitou agrupar 20 variáveis em sete fatores, que explicam simultaneamente $81 \%$ da variância total da amostra. Esse agrupamento permitiu a interpretabilidade das relações existentes entre elas, ou seja, as suas interdependências e a influência no crescimento e desenvolvimento da sojicultura e impacto na competitividade da cadeia produtiva da soja em Moçambique. Por conseguinte, com a análise de agrupamento foi possível agrupar os sojicultores em quatro clusters: os que estão mais engajados na evolução da sojicultura moçambicana e recebem mais contribuição de políticas públicas e privadas; aqueles com média evolução produtiva e com menos apoio institucional; os que tiveram menos evolução da produtividade e receberam mais apoio e os que não tiveram nenhum apoio. Portanto, foi possível concluir que a ação de políticas públicas e privadas nas regiões em estudo, proporcionaram melhores índices produtivos da sojicultura.

Palavras-chaves: Sojicultura. Cadeia produtiva. Competitividade. Moçambique.

\section{COMPETITIVENESS OF SOJICULTURE IN MOZAMBIQUE: AN ANALYSIS BASED ON THE EXPERIENCE OF LOCAL PRODUCERS}

Abstract: Soy farming, on a global level, is among the economic activities that, in the last decades, presented more expressive growth. Therefore, the objective was to analyze the competitiveness of Mozambican soybean farming using multivariate statistical techniques, based on the theory of systemic competitiveness. The survey used primary data, obtained from January to March 2018, in Mozambique. The sample consisted of 120 soybean growers, in which the sampling technique was nonprobabilistic and the choice of producers was based on the "snowball" technique. Descriptive statistics and multivariate statistics (Exploratory Factor Analysis and Cluster Analysis) were used as a reference for data compilation and treatment. The factor analysis made it possible to group 20 variables into seven factors, which

\footnotetext{
${ }^{1}$ Universidade Zambeze, Departamento de Economia Agrária e Desenvolvimento Rural, Cidade de Mocuba - Zambézia, Moçambique, ricardinajaneque17@gmail.com, https://orcid.org/0000-0002-51418496

${ }^{2}$ Universidade Federal de Santa Maria, Departamento de Ciências Econômicas, Palmeira das Missões - RS, Brasil, nilson.costa@ufsm.br, https://orcid.org/0000-0003-2000-1521
} 
simultaneously explain $81 \%$ of the total sample variance. This grouping allowed for the interpretability of the existing relations between them, that is, their interdependencies and the influence on the growth and development of soybean and impact on the competitiveness of the soy production chain in Mozambique. Therefore, with the cluster analysis it was possible to group soybean farmers into four clusters: those who are most engaged in the evolution of Mozambican soybean and receive the most contribution from public and private policies; those with medium productive evolution and withless institutional support; those who had less productivity growth and received more support and those who had no support. Therefore, it was possible to conclude that the action of public and private policies in the studied regions provided better soybean production indexes.

Keywords: Soybean. Productive Chain. Competitiveness. Mozambique.

\section{COMPETITIVIDAD DE LA SOJICULTURA EN MOZAMBIQUE: UN ANÁLISIS BASADO EN LA EXPERIENCIA DE PRODUCTORES LOCALES}

Resumen: La cultura de la soja, a nivel mundial, se encuentra entre las actividades económicas que, en las últimas décadas, presentaron un crecimiento más expresivo. Por lo tanto, el objetivo era analizar la competitividad del cultivo de soja de Mozambique utilizando técnicas estadísticas multivariadas, basadas en la teoría de la competitividad sistémica. La encuesta utilizó datos primarios, obtenidos de enero a marzo de 2018, en Mozambique. La muestra consistió en 120 productores de soja, en los cuales la técnica de muestreo no era probabilística y la elección de los productores se basó en la técnica de "bola de nieve". Las estadísticas descriptivas y las estadísticas multivariadas (Análisis factorial exploratorio y Análisis de conglomerados) se utilizaron como referencia para la compilación y el tratamiento de datos. El análisis factorial permitió agrupar 20 variables en siete factores, que explican simultáneamente el $81 \%$ de la varianza total de la muestra. Esta agrupación permitió la interpretación de las relaciones existentes entre ellos, es decir, sus interdependencias y la influencia en el crecimiento y desarrollo de la soja y el impacto en la competitividad de la cadena de producción de soja en Mozambique. Por lo tanto, con el análisis de conglomerados, fue posible agrupar a los productores de soja en cuatro conglomerados: aquellos que están más involucrados en la evolución de la soja mozambiqueña y reciben la mayor contribución de las políticas públicas y privadas; aquellos con evolución productiva media y con menos apoyo institucional; aquellos que tuvieron un menor crecimiento de la productividad y recibieron más apoyo y aquellos que no tuvieron ningún apoyo. Por lo tanto, fue posible concluir que la acción de las políticas públicas y privadas en las regiones estudiadas proporcionó mejores índices de producción de soja.

Palabras clave: Producción de soja. Cadena productiva. Competitividad. Mozambique.

\section{Introdução}

A sojicultura, em nível global, está entre as atividades econômicas que, nas últimas décadas, apresentaram crescimentos mais expressivos. Isso pode ser atribuído a diversos fatores, dentre os quais: desenvolvimento e estruturação de um sólido mercado internacional relacionado com o comércio de produtos do complexo grão, farelo e óleo de soja; consolidação da oleaginosa como importante fonte de 
proteína e óleo vegetal, especialmente para atender demandas crescentes dos setores ligados à produção de produtos de origem animal e ampliação da oferta de alimentos processados e ultraprocessados, destinados diretamente para a população humana; geração e oferta de tecnologias, que viabilizaram a expansão da exploração sojícola para diversas regiões do mundo (EMBRAPA, 2014; COSTA et al., 2014).

Em Moçambique, o mercado da soja é relativamente novo, tendo apresentado um rápido crescimento, com a produção dominada por pequenos agricultores ${ }^{3}$ que estão emergindo para agricultores comerciais ${ }^{4}$, e com uma margem de lucro considerável para crescimento da produção (PEREIRA; YAN, 2012). O consumo de frango em rápido crescimento é o principal determinante da crescente procura de farelo de soja (TECHNOSERVE BUSINESS SOLUTION TO POVERTY, 2011).

Um dos aspetos mais ressaltantes do sucesso da soja é o seu potencial de mudança estrutural na economia moçambicana, criando oportunidades para uma mobilidade ascendente no seio da comunidade agrícola. Verifica-se também que unidades de produção emergentes usam a soja como trampolim para aumentar o tamanho da exploração agrícola para maiores operações comerciais (WALKER; CUNGUARA, 2016).

$\mathrm{Na}$ discussão atual, a capacidade de se articular cadeias produtivas competitivas depende sobremaneira de sua inserção complementar as suas congêneres em nível local. As transformações que decorrem do processo de globalização, associadas a outras, tecnológicas e institucionais, impactam profundamente as formas de organização e as premissas de desenvolvimento de cada país ou região. As estratégias dos grandes agentes econômicos implicam em escolhas de regiões que se integram ou que possuem dificuldades para se convergirem, o que vem redefinindo hierarquias e dinâmicas de crescimento econômico de territórios em toda aldeia global (RITA; JUNIOR, 2005).

Destaca-se, também, que a inserção dos empreendimentos no mundo dos negócios em nível global requer a percepção, por parte das instituições públicas e privadas dos Estados, de que a competição não só está mais incitada como as

\footnotetext{
${ }^{3}$ Pequenos agricultores classificados como aqueles que possuem área cultivada não irrigada menor que 10 ha (CAP, 2009).

4 Aos "agricultores emergentes" comerciais referem-se aqueles que têm pequenas e médias explorações (SMART; HANLON, 2014). São consideradas médias, as explorações com área cultivada não irrigada maior ou igual a 10 ha e menores que 50 ha (CAP, 2009). Segundo O Censo Agropecuário 2009/10 não há absoluta distinção entre explorações comerciais e não comerciais. Mesmo as pequenas explorações podem vender os seus produtos.
} 
vantagens competitivas passam a depender cada vez mais da interação sinérgica entre as dimensões empresarial, estrutural e sistêmica. A primeira dimensão tem a ver com a capacidade empreendedora dos agentes econômicos; a segunda, com a infraestrutura (logística) local/regional e a terceira está relacionada às principais tendências em níveis nacional e internacional (SUDENE, 2003).

Isto posto, o estudo teve por objetivo analisar a competitividade da sojicultura moçambicana a partir de técnicas de estatística multivariada. Para tal, a teoria da competitividade sistêmica contribuiu para a escolha das variáveis e delineamento da pesquisa. Por sua vez, o tratamento dos dados foi realizado a partir das técnicas de estatística multivariada, Análise Fatorial Exploratória (AFE) e Análise de Conglomerados (AC).

O artigo foi estruturado em quatro secções, em que, a primeira compreende esta introdução e a segunda apresenta-se o referencial metodológico onde explicase detalhadamente os procedimentos utilizados para responder o objetivo. Por conseguinte, na terceira secção estão apresentados os resultados encontrados durante a pesquisa e as discussões, em que são apresentados em duas partes, sendo a primeira referente a técnica multivariada da análise fatorial e a segunda referente a análise de agrupamentos. Por fim, na quarta secção estão apresentadas as considerações finais.

\section{Desenvolvimento}

Quanto aos objetivos, esta pesquisa pode ser classificada, conforme Gil (2002), como exploratória. Pois, segundo o autor, têm como principal finalidade desenvolver, esclarecer e modificar conceitos e ideias, tendo em vista a formulação de problemas mais precisos ou hipóteses pesquisáveis para estudos posteriores. $\mathrm{E}$ quanto aos procedimentos técnicos, como uma pesquisa quantitativa, por se centrar na objetividade.

\section{Área de estudo}

A área de estudo corresponde à região composta pelos Distritos de Ribáuè, Malema, Gurué, Namarroe, Alto Molócuè, Sussundenga, Bárue, Macate, Manica, Tsangano e Angónia, localizadas em Moçambique, Sudeste do Continente Africano.

Figura 1 - Área de estudo 


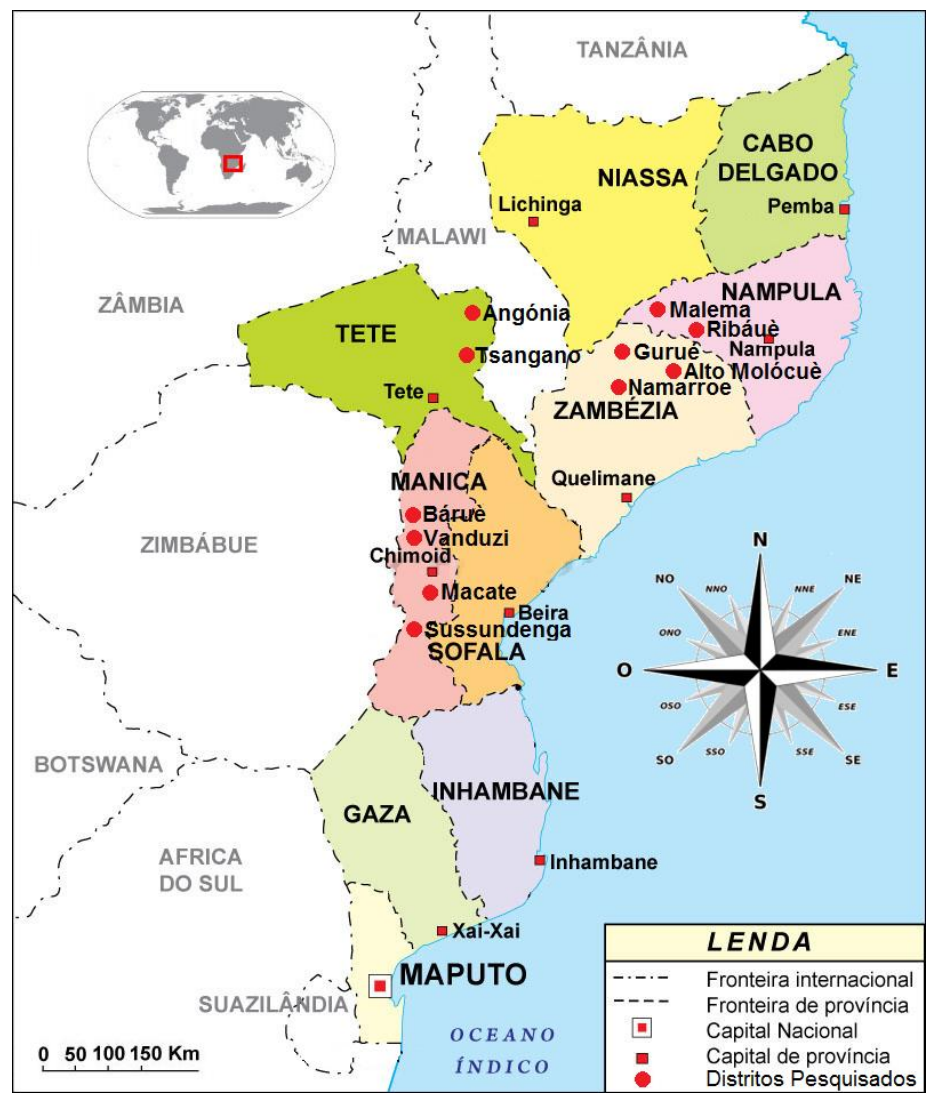

Fonte: Elaboração própria com base em MAPAS DO MUNDO (2014)

Esta região é caracterizada por concentrar cerca de $62,17 \%$ da produção de soja de Moçambique, que foi de aproximadamente 35.247 toneladas no ano de 2016, conforme pode ser observado na Tabela 01.

Tabela 01- Produção de soja em Moçambique: 2016

\begin{tabular}{lccc}
\hline \multicolumn{1}{c}{ Distritos } & $\begin{array}{c}\text { Produção } \\
\text { (toneladas) }\end{array}$ & $\begin{array}{c}\text { Frequência } \\
\text { Relativa }\end{array}$ & $\begin{array}{c}\text { Frequência } \\
\text { Acumulada }\end{array}$ \\
\hline Angónia & $8.325,00$ & $23,62 \%$ & $23,62 \%$ \\
Gurué & $7.900,00$ & $22,41 \%$ & $46,03 \%$ \\
\hline Báruè & $1.750,00$ & $4,96 \%$ & $51,00 \%$ \\
\hline Tsangano & $1.495,00$ & $4,24 \%$ & $55,24 \%$ \\
\hline Alto Molócuè & $1.250,00$ & $3,55 \%$ & $58,78 \%$ \\
\hline Sussundenga, Macate, Manica & 555,00 & $1,57 \%$ & $60,36 \%$ \\
\hline Namarroe & 550,00 & $1,56 \%$ & $61,92 \%$ \\
\hline Malema & 55,00 & $0,16 \%$ & $62,08 \%$ \\
\hline Ribáuè & 31,87 & $0,09 \%$ & $62,17 \%$ \\
\hline Outros & $13.335,42$ & $37,83 \%$ & $\mathbf{1 0 0 , 0 0 \%}$ \\
\hline Produção total do País (t) & $\mathbf{3 5 . 2 4 7 , 3 0}$ & $\mathbf{1 0 0 , 0 0 \%}$ & \\
\hline
\end{tabular}

Fonte: TechnoServe Mozambique (2016).

\section{Dados}

Foram utilizados dados primários, obtidos em pesquisa de campo realizada entre os meses de janeiro e março de 2018. Os produtores de soja entrevistados e que responderam questionários foram escolhidos a partir da técnica de amostragem "bola de neve". 
O instrumento de coleta de dados foi um questionário estruturado, que pela sua popularidade e importância de utilização em pesquisas sociais possibilitam medir variáveis e descrever situações de forma ordenada e consistente.

Os questionários foram organizados em quatro grandes blocos, com questões relacionadas às dimensões da Competitividade Sistêmica. As variáveis selecionadas para o tratamento de dados, por meio das duas técnicas multivariadas, encontramse descritas no quadro a seguir.

Quadro 1- Variáveis utilizadas para análise multivariada de dados.

\begin{tabular}{|c|c|}
\hline $\begin{array}{l}\text { Designação } \\
\text { da variável }\end{array}$ & Descrição da variável \\
\hline V1 & Vantagem locacional inerente a Proximidade com os fornecedores de insumos \\
\hline V2 & Vantagem locacional inerente a Proximidade com os clientes/consumidores \\
\hline V3 & $\begin{array}{l}\text { Vantagem locacional inerente a Infraestrutura física (energia, transporte, } \\
\text { comunicações) }\end{array}$ \\
\hline V4 & $\begin{array}{l}\text { Vantagem locacional inerente a Disponibilidade de serviços técnicos } \\
\text { especializados }\end{array}$ \\
\hline V5 & $\begin{array}{l}\text { Vantagem locacional inerente a Existência de programas de apoio e promoção a } \\
\text { produção }\end{array}$ \\
\hline V6 & $\begin{array}{l}\text { A contribuição de associações, cooperativas locais e ONGs no tocante a } \\
\text { Disponibilização de informações (referentes a matéria-prima, equipamento, } \\
\text { assistência técnica, consultoria, etc.) }\end{array}$ \\
\hline V7 & $\begin{array}{l}\text { A contribuição de associações, cooperativas locais e ONGs no tocante a } \\
\text { Promoção de ações cooperativas }\end{array}$ \\
\hline V8 & $\begin{array}{l}\text { A contribuição de associações, cooperativas locais e ONGs no tocante a } \\
\text { Promoção de ações dirigidas a capacitação tecnológica de produtores }\end{array}$ \\
\hline V9 & Evolução da produtividade nos últimos 3 anos \\
\hline V10 & Evolução do Custo de produção nos últimos 3 anos \\
\hline V11 & Evolução do faturamento nos últimos 3 anos \\
\hline V12 & O nível de ligação com o Governo \\
\hline V13 & A importância atribuída para o papel das ONGs \\
\hline V14 & A importância atribuída para o papel do Governo \\
\hline V15 & A relevância do preço de insumo na seleção de fornecedores \\
\hline V16 & A relevância da proximidade dos fornecedores na seleção de fornecedores \\
\hline V17 & A importância atribuída para o Acesso ao crédito \\
\hline V18 & A importância atribuída para o Escoamento de produto \\
\hline V19 & $\begin{array}{l}\text { O grau de importância de Aumentar a área plantada de soja como principal } \\
\text { objetivo }\end{array}$ \\
\hline V20 & $\begin{array}{l}\text { O grau de importância de Aumentar a produtividade da soja como principal } \\
\text { objetivo }\end{array}$ \\
\hline
\end{tabular}

Fonte: Dados da pesquisa de campo (2018)

Considerando que estas variáveis foram obtidas a partir da aplicação de questionários, utilizou-se o alfa de Cronbach (HAIR, 2009) para avaliar a confiabilidade do constructo utilizado para a coleta de dados da pesquisa.

\section{Técnicas de tratamento dos dados}

Para o tratamento dos dados foram utilizadas técnicas de estatística descritiva (distribuição de frequência e as medidas de tendência central) e de estatística 
multivariada (Análise Fatorial Exploratória e Análise de Conglomerados). As técnicas foram selecionadas pela capacidade de contribuir e responder os objetivos da pesquisa, conforme recomendação metodológica de Hair (2009) e Costa et al (2017).

As técnicas de estatística descritiva foram aplicadas na análise inicial dos dados para organizar, resumir e descrever os aspectos importantes de um conjunto de características observadas ou comparar tais características entre dois ou mais conjuntos (REIS; REIS, 2002). Os dados foram sintetizados em tabelas, gráficos e também em medidas de síntese como porcentagens, índices e médias.

A análise multivariada foi empregada, na presente pesquisa, para reduzir ou simplificar a estrutura de um fenômeno com o objetivo de possibilitar sua interpretação com a menor perda de informação possível; selecionar ou agrupar variáveis segundo suas características; investigar relações de dependência ou independência entre variáveis e as naturezas dessas relações (JOHNSON; WICHERN, 1992; FÁVERO et al., 2009). Para tal, utilizou-se a Análise Fatorial Exploratória e Análise de Agrupamentos.

\section{Análise Fatorial Exploratória}

Impõem-se como pioneiros de estudos sobre análise fatorial Karl Pearson (1901), Charles Spearman (1904) e Louis Thurstone (1931). Entretanto, Corrar, Paulo e Dias Filho (2017) definem a análise fatorial como "uma técnica estatística que busca, através da avaliação de um conjunto de variáveis, a identificação de dimensões de variabilidade comuns existentes em um conjunto de fenômenos; o intuito é desvendar estruturas existentes, mas que não observáveis diretamente".

Portanto, o objetivo da análise fatorial é o agrupamento de variáveis que apresentam alto grau de correlação, de modo a facilitar o entendimento e análise das mesmas no estudo (FÁVERO et al., 2009; HAIR et al., 2009; CORRAR, PAULO, DIAS FILHO, 2017). Em outras palavras, a análise fatorial avalia a possibilidade de agrupar $i$ variáveis $\left(\mathrm{X}_{1}, \mathrm{X}_{2}, \mathrm{X}_{3} \ldots \mathrm{X}_{i}\right)$ em número menor de $j$ fatores $\left(\mathrm{F}_{1}, \mathrm{~F}_{2}, \mathrm{~F}_{3} \ldots \mathrm{F}_{j}\right)$. Podendo ser apresentado na seguinte forma matemática:

$X_{i}=\alpha_{i 1} F_{1}+\alpha_{i 2} F_{2}+\alpha_{i 3} F_{3}+\ldots+\alpha_{i j} F_{j}+\varepsilon_{i}$ Equação (1)

Onde: $X_{i}$ são as variáveis observáveis padronizadas; $\alpha_{i}$ são as cargas fatoriais; $F_{j}$ são os fatores comuns não relacionados entre si; $\varepsilon_{i}$ é um erro que 
representa a parcela de variação da variável $i$ que é exclusiva dela e não pode ser explicada por um fator nem por outra variável do conjunto analisado.

Por conseguinte, os fatores podem ser estimados por uma combinação linear das variáveis originais, como apresentado na equação 2 :

$$
F_{j}=\omega_{j 1} X_{1}+\omega_{j 2} X_{2}+\omega_{j 3} X_{3}+\ldots+\omega_{j i} X_{i}+\varepsilon_{i}
$$

Em que: $F_{j}$ são os fatores comuns não relacionados, $\omega_{j i}$ são os coeficientes dos escores fatoriais (é um número resultante da multiplicação dos coeficientes $\omega_{j i}$ pelo valor das variáveis originais), Xi são as variáveis originais do estudo.

Para aferir a qualidade das correlações entre as variáveis e a adequação da amostra foram utilizados os testes de Kaiser-Meyer-Olkin (KMO) e de Esfericidade de Bartlett. Para o KMO, resultados superiores a 0,6 são considerados aceitáveis e no caso de teste de Esfereciade de Barlett, espera-se significância estatística ao nível de $1 \%$ de probabilidade (HAIR, 2009).

A Matriz Anti-Imagem e as Comunalidades foram utilizadas como critério para escolher as variáveis que permaneceram nos fatores.

A extração dos fatores foi procedida com base no método de componentes principais e para a rotação dos mesmos, empregou-se o método VARIMAX, por ser o método que, conforme Corrar, Paulo e Dias Filho (2017), minimiza a ocorrência de uma variável possuir altas cargas fatoriais para diferentes fatores.

\section{Análise de conglomerados}

A análise de conglomerados tem por finalidade primária agregar objetos com base nas características que eles possuem (HAIR et al., 2006, 2009; MINGOTI, 2007; CORRAR, PAULO, DIAS FILHO, 2017).

Neste estudo, a finalidade da técnica foi identificar a formação de grupos através da análise de semelhanças e diferenças existentes entre suas características, ou seja, que sejam homogêneos internamente, heterogêneos externamente e mutuamente exclusivos (FÁVERO et al., 2009).

Nessa perspectiva, a técnica foi empregada na presente pesquisa para classificar os sojicultores moçambicanos conforme as diversas características/variáveis de ótica da competividade sistêmica. 
Escolheu-se a distância euclidiana quadrática (dij2) para medir a similaridade entre os sojicultores. Ela mede o raio entre duas observações ( $i$ e $f$ ) para todas as $p$ variáveis do modelo (FÁVERO et al., 2009), e pode ser expressa da seguinte forma.

$d^{2}{ }_{i j}=\sum_{k=1}^{p}\left(X_{i k}-X_{j k}\right)^{2}$

(Equação 3)

O método de agrupamento hierárquico foi utilizado para fazer o cronograma de aglomeração e o dendrograma. O número de grupos de produtores formados foi escolhido com base da regra da parada (HAIR, 2009). A similaridade entre os agrupamentos foi definida pelo algoritmo ligação média (entre grupos).

O método não hierárquico (algoritmo K-médias) foi utilizado para identificar qual ou quais das variáveis foram mais determinantes na separação dos clusters.

\section{Resultados e discussões}

Os resultados estão apresentados em três subseções. $\mathrm{Na}$ primeira, apresenta-se o perfil dos produtores e da sojicultura moçambicana e nas seguintes, a análise da competitividade, com base nas técnicas de estatística multivariada.

\section{Características dos sojicultores moçambicanos}

Entre os produtores de soja entrevistados, houve maior proporção de sojicultores do sexo masculino em relação a do sexo feminino, com predominância de sojicultores com 30-50 anos, sendo 19 anos a idade mínima e 73 anos a idade máxima (Tabela 2). A maioria vive maritalmente (82\%), ou seja, vivem casados mas não oficialmente, entretanto, 65\% possuem 6-10 membros, havendo também uma superioridade de famílias com 1-5 membros. Quanto ao nível de escolaridade, 75\% dos sojicultores possuem nível primário.

Tabela 02 - Perfil dos sojicultores moçambicanos entrevistados (2018)

\begin{tabular}{cccc}
\hline & & Frequência & $\%$ \\
\hline Sexo & Feminino & 38 & 31 \\
& Masculino & 86 & 69 \\
\hline Idade & $19-29$ & 14 & 11 \\
& $30-40$ & 44 & 35 \\
& $41-50$ & 33 & 27 \\
& $51-60$ & 22 & 18 \\
& $61-70$ & 4 & 3 \\
& $71-80$ & 3 & 2 \\
& Não sabe & 4 & 3 \\
\hline
\end{tabular}




\begin{tabular}{cccc}
\hline Estado civil & Casado & 12 & 10 \\
& Convivência Marital & 102 & 82 \\
& Solteiro & 7 & 6 \\
& Viúvo & 3 & 2 \\
\hline Nível de & Não-alfabetizado & 7 & 6 \\
escolaridade & Primário & 93 & 75 \\
& Secundário incompleto & 21 & 17 \\
& Secundário completo & 3 & 2 \\
\hline Membros & $1-5$ & 45 & 36 \\
familiares & $6-10$ & 65 & 52 \\
& $11-15$ & 12 & 10 \\
& $16-20$ & 2 & 2 \\
\hline
\end{tabular}

Fonte: Dados da pesquisa de campo (2018).

Quanto ao período de início da atividade sojícola, a maioria dos produtores (46\%) encontram-se entre 1-5 anos, existindo alguns cultivando a mais de dez anos (19\%), o que demonstra que esta é uma atividade recente no país.

Cerca de $29 \%$ dos produtores de soja teve área inicial plantada de 0,5 hectare, $27 \%$ teve 1,0 ha e $14 \%$ teve 2,0 ha. Apesar da soja estar sendo cultivada a mais de dez anos, $22 \%$ e $21 \%$ dos produtores, atualmente, detém, respectivamente, 2ha e 1ha de área plantada com soja. Portanto, além de ser uma atividade nova, está restrita a pequenas frações de áreas.

No que concerne à produtividade de soja obtida pelos entrevistados, os resultados variaram bastante, tendo-se, de 0,125 t/hectare a $4 \mathrm{t} / \mathrm{ha}$, a produtividade menor e maior, respectivamente. Portanto, as produtividades mais destacadas encontram-se no intervalo de $0,125-1,5 \mathrm{t} / \mathrm{ha}$.

\section{Análise Multivariada da Competitividade da Sojicultura moçambicana}

\section{Análise Fatorial}

A análise fatorial foi utilizada para analisar o conjunto de variáveis que compõem o questionário da pesquisa de campo, com intuito de identificar as dimensões de variabilidade comuns existentes.

O modelo da análise fatorial foi composto pelas variáveis apresentadas na Tabela 3, tendo sido validado pelo coeficiente Alfa de Cronbach $\alpha=0,813$, considerado por Hair et al. (2009) adequado para pesquisas exploratórias. Por conseguinte, a consistência das variáveis foi verificada através do coeficiente Alfa de Cronbach, em que quase todas apresentaram valores superiores a 0,700. A estatística descritiva demonstrou que as variáveis V3, V4, V5, V2, V1, V8 e V7 apresentaram maiores médias no conjunto das variáveis.

Tabela 03 - Variáveis utilizadas na análise fatorial de dados. 


\begin{tabular}{|c|c|c|c|c|}
\hline $\begin{array}{l}\text { Designação } \\
\text { da variável }\end{array}$ & Descrição da variável & Média & $\mathbf{N}$ & $\begin{array}{c}\text { Alfa de } \\
\text { Cronbach }\end{array}$ \\
\hline $\mathrm{V} 1$ & $\begin{array}{l}\text { Vantagem locacional inerente a Proximidade com os } \\
\text { fornecedores de insumos }\end{array}$ & 4,586 & 116 & 0,807 \\
\hline V2 & $\begin{array}{l}\text { Vantagem locacional inerente a Proximidade com os } \\
\text { clientes/consumidores }\end{array}$ & 4,629 & 116 & 0,807 \\
\hline V3 & $\begin{array}{l}\text { Vantagem locacional inerente a Infraestrutura física } \\
\text { (energia, transporte, comunicações) }\end{array}$ & 4,724 & 116 & 0,809 \\
\hline V4 & $\begin{array}{l}\text { Vantagem locacional inerente a Disponibilidade de } \\
\text { serviços técnicos especializados }\end{array}$ & 4,707 & 116 & 0,807 \\
\hline V5 & $\begin{array}{l}\text { Vantagem locacional inerente a Existência de } \\
\text { programas de apoio e promoção a produção }\end{array}$ & 4,707 & 116 & 0,805 \\
\hline V6 & $\begin{array}{l}\text { A contribuição de associações, cooperativas locais e } \\
\text { ONGs no tocante a Disponibilização de informações } \\
\text { (referentes a matéria-prima, equipamento, } \\
\text { assistência técnica, consultoria, etc.) }\end{array}$ & 4,216 & 116 & 0,799 \\
\hline V7 & $\begin{array}{l}\text { A contribuição de associações, cooperativas locais e } \\
\text { ONGs no tocante a Promoção de ações } \\
\text { cooperativas }\end{array}$ & 4,138 & 116 & 0,788 \\
\hline V8 & $\begin{array}{l}\text { A contribuição de associações, cooperativas locais e } \\
\text { ONGs no tocante a Promoção de ações dirigidas a } \\
\text { capacitação tecnológica de produtores }\end{array}$ & 4,233 & 116 & 0,792 \\
\hline V9 & Evolução da produtividade nos últimos 3 anos & 3,828 & 116 & 0,807 \\
\hline V10 & Evolução do Custo de produção nos últimos 3 anos & 3,483 & 116 & 0,817 \\
\hline V11 & Evolução do faturamento nos últimos 3 anos & 3,784 & 116 & 0,808 \\
\hline V12 & O nível de ligação com o Governo & 3,759 & 116 & 0,807 \\
\hline V13 & A importância atribuída para o papel das ONGs & 4,043 & 116 & 0,801 \\
\hline V14 & A importância atribuída para o papel do Governo & 3,957 & 116 & 0,805 \\
\hline V15 & $\begin{array}{l}\text { A relevância do preço de insumo na seleção de } \\
\text { fornecedores }\end{array}$ & 3,052 & 116 & 0,82 \\
\hline V16 & $\begin{array}{l}\text { A relevância da proximidade dos fornecedores na } \\
\text { seleção de fornecedores }\end{array}$ & 3,052 & 116 & 0,817 \\
\hline V17 & A importância atribuída para o Acesso ao crédito & 4,69 & 116 & 0,813 \\
\hline V18 & $\begin{array}{l}\text { A importância atribuída para o Escoamento de } \\
\text { produto }\end{array}$ & 4,81 & 116 & 0,812 \\
\hline V19 & $\begin{array}{l}\text { O grau de importância de Aumentar a área plantada } \\
\text { de soja como principal objetivo }\end{array}$ & 4,905 & 116 & 0,817 \\
\hline V20 & $\begin{array}{l}\text { O grau de importância de Aumentar a produtividade } \\
\text { da soja como principal objetivo }\end{array}$ & 4,957 & 116 & 0,816 \\
\hline
\end{tabular}

Fonte: Dados da pesquisa de campo (2018).

A matriz de correlação apresentou $52 \%$ de variáveis significativas a $5 \%$ e $23 \%$ de correlações médias e fortes $(\geq 0,300)$ entre as variáveis, confirmando de forma preliminar, a adequação da amostra. Ademais, o determinante da mesma matriz confirmou a adequação da matriz de dados por apresentar valor diferente de zero (2,00E-006).

$\mathrm{O}$ valor de $\mathrm{KMO}$ da amostra foi 0,708 , encontrando-se acima do intervalo crítico. Da mesma forma, o teste de esfericidade de Bartlett apresentou o valor 1410,549 e estatística menor que 0,01, sugerindo que as correlações de Pearson entre as variáveis são estatisticamente diferentes de zero. Assim sendo, os dois testes sugerem que a amostra é adequada para extração de fatores e que é apropriada a realização da análise fatorial. 
A adequação de cada variável na amostra foi confirmada pela matriz antiimagem, em que observa-se que 18 variáveis apresentaram valores de MSA (Medidas de adequação de amostragem) superiores a 0,500 e apenas duas variáveis (V19 e V20) apresentaram valores abaixo do recomendado. Portanto, devido a centralidade das variáveis no micronível da competividade sistêmica da sojicultora moçambicana e pela importância das variáveis no modelo (Alfa de Cronbach 0,805 e 0,82, respetivamente), não serão excluídas do modelo. Por outro lado, a maior parte das variáveis apresentaram valores de comunalidade acima de 0,800 (Tabela 4).

Depois de confirmada a adequação da amostra para análise fatorial, seguiuse com a extração de fatores. O método de extração foi Componentes Principais e o método de rotação foi Varimax. Entretanto, a rotação dos fatores possibilitou a extração de sete fatores, sendo que todos apresentam autovalores maiores que um e explicam simultaneamente $81 \%$ da variância total da amostra. O primeiro fator apresenta autovalor de 5,683 carregando cerca de $20 \%$ da variância e 2,748 (13,209\%); 1,855 (10,601\%); 1,752 (9,571\%); 1,567 (9,284\%); 1,307 (8,993\%); $1,229(\mathbf{8}, 931 \%)$, respetivamente, o segundo, terceiro, quarto, quinto, sexto e sétimo fator.

Tabela 04 - Matriz de componente extrativa rotacionada e comunalidades

\begin{tabular}{|c|c|c|c|c|c|c|c|c|}
\hline \multirow{2}{*}{ Variáveis } & \multicolumn{7}{|c|}{ Componentes (Fatores) } & \multirow{2}{*}{$\begin{array}{c}\text { Comuna- } \\
\text { lidades }\end{array}$} \\
\hline & 1 & 2 & 3 & 4 & 5 & 6 & 7 & \\
\hline V4 & 0,916 & 0,115 & 0,027 & 0,136 & $-0,064$ & 0,087 & $-0,018$ & 0,884 \\
\hline V5 & 0,89 & 0,184 & 0,002 & 0,032 & 0,006 & 0,005 & 0,109 & 0,838 \\
\hline V3 & 0,817 & 0,046 & $-0,002$ & 0,15 & $-0,064$ & 0,307 & $-0,019$ & 0,791 \\
\hline V2 & 0,816 & 0,152 & 0,013 & 0,038 & 0,05 & 0,175 & $-0,026$ & 0,724 \\
\hline V1 & 0,789 & 0,246 & 0,127 & 0,011 & 0,092 & 0,149 & $-0,051$ & 0,732 \\
\hline V7 & 0,191 & 0,899 & 0,074 & 0,026 & $-0,059$ & 0,076 & 0,04 & 0,862 \\
\hline V6 & 0,239 & 0,848 & 0,22 & 0,17 & 0,013 & 0,118 & 0,021 & 0,868 \\
\hline V8 & 0,257 & 0,786 & 0,075 & 0,222 & 0,149 & 0,039 & $-0,002$ & 0,763 \\
\hline V9 & 0,094 & 0,243 & 0,819 & $-0,03$ & 0,071 & $-0,075$ & 0,121 & 0,764 \\
\hline V10 & $-0,129$ & 0,049 & 0,81 & 0,18 & $-0,104$ & 0,133 & $-0,103$ & 0,747 \\
\hline V11 & 0,163 & 0,003 & 0,794 & 0,081 & 0,22 & $-0,13$ & 0,152 & 0,751 \\
\hline V12 & 0,202 & 0,018 & 0,152 & 0,856 & 0,023 & $-0,107$ & 0,033 & 0,81 \\
\hline V14 & 0,083 & 0,214 & 0,14 & 0,797 & $-0,024$ & 0,151 & 0,085 & 0,737 \\
\hline V13 & $-0,026$ & 0,469 & $-0,151$ & 0,597 & 0,162 & 0,167 & 0,153 & 0,677 \\
\hline V15 & 0,031 & 0,035 & 0,054 & 0,001 & 0,926 & 0,02 & $-0,154$ & 0,887 \\
\hline V16 & $-0,028$ & 0,053 & 0,083 & 0,063 & 0,923 & $-0,016$ & 0,12 & 0,88 \\
\hline V18 & 0,223 & 0,154 & $-0,026$ & 0,034 & 0,043 & 0,884 & $-0,041$ & 0,86 \\
\hline V17 & 0,328 & 0,062 & $-0,035$ & 0,073 & $-0,04$ & 0,858 & 0,027 & 0,857 \\
\hline V19 & 0,089 & 0,008 & 0,041 & 0,03 & $-0,075$ & 0,007 & 0,923 & 0,868 \\
\hline V20 & $-0,099$ & 0,062 & 0,091 & 0,135 & 0,047 & $-0,022$ & 0,892 & 0,839 \\
\hline \% de variância & 20,116 & 13,209 & 10,601 & 9,571 & 9,284 & 8,993 & 8,931 & \\
\hline Método de Extrac & $\begin{array}{l}\text { Análise } \\
\text { Varimax }\end{array}$ & $x_{0}$ & alizaçã & ncipal. & & & & \\
\hline
\end{tabular}


O primeiro fator explicou $20,116 \%$ da variância total, foi denominado Vantagem Locacional porque reuniu variáveis relacionadas as vantagens que as localizações proporcionam em relação a disponibilidade de serviços técnicos especializados, a existência de programas de apoio e promoção a produção, a proximidade com os clientes/consumidores, a infraestrutura física (energia, transporte, comunicações) e a proximidade com os fornecedores de insumos. A disponibilidade de serviços técnicos especializados em várias regiões do mundo proporcionam desenvolvimento produtivos e ganhos competitivos, o que incrementase com a presença de programas de apoio e promoção da produção. Em Moçambique, essas duas variáveis, geralmente, atuam em simultâneo. Ou seja, a expansão do uso da tecnologia é creditada pelas parcerias público-privadas, que financiam maquinários e outras tecnologias para o desenvolvimento das atividades agrícolas.

Entretanto, a presença de programas de apoio e promoção a produção e a disponibilidade de serviços técnicos especializados constituem aspectos importantes para a expansão e desenvolvimento da sojicultura em Moçambique. A proximidade com clientes/consumidores e fornecedores de insumo também se tornam importantes sobretudo por reduzirem os custos de transporte e facilidade na especulação dos preços (pesquisa de mercado).

A infraestrutura física (energia, transporte, comunicações) é um dos fatores considerados importantes para o desenvolvimento de diversas atividades, especialmente a agrícola. Para que as variáveis acima sejam alcançadas com sucesso torna-se necessário, a priori, a existência de uma boa infraestrutura. Pois, a cadeia produtiva de soja por envolver fluxos de bens e serviços, a existência de transporte, estradas em boas condições e comunicação tornam-se necessários para expansão e consolidação da atividade sojícola. Outro aspecto a considerar é o afirmado por Esser et al. (1996) que a política de infraestrutura física deve assegurar que as vantagens competitivas, adquiridas no processo produtivo, não sejam anuladas no processo de distribuição, por deficiências nos canais de distribuição, como estradas ruins, por exemplo.

Portanto, o fator Vantagem Locacional englobou variáveis que se enquadram no mesonível da competitividade sistêmica. Essas variáveis impulsionam políticas de estímulo à produção e disseminação de tecnologia e melhorias de infraestrutura, o que contribui para a criação de vantagens competitivas nacionais. 
O segundo fator, que explicou 13,209\% da variância total, foi designado Contribuição de associações, cooperativas locais e ONGs, por reunir variáveis relacionadas a contribuição dessas instituições no incremento do desempenho produtivo dos sojicultores. Foi formado por três variáveis: o apoio dessas instituições na disponibilização de informações (referentes a matéria-prima, equipamento, assistência técnica, consultoria, etc.), na promoção de ações cooperativas e na promoção de ações dirigidas a capacitação tecnológica de produtores. Salienta-se que, além destas variáveis, a variável importância atribuída ao papel das ONGs, apresentou carga fatorial forte para este fator embora ter-se constituído no fator quatro, ou seja, esta variável também contribui para o segundo fator.

A contribuição institucional é de tamanha importância na consolidação das atividades agropecuárias. Especificamente na sojicultura moçambicana, o apoio destas entidades na expansão do cultivo e no desenvolvimento de todas atividades sojícolas é considerável.

Sob ponto de vista da competitividade sistêmica as instituições devem procurar resolver problemas dos produtores, enfatizando políticas de apoio, estímulo e avaliação de atividades com vista ao desenvolvimento das atividades e à competitividade (ESSER et al., 1994). Entretanto, as variáveis que compõem este fator subsidiam estas premissas. Na medida em que estas instituições capacitam ou promovem capacitação aos produtores para o uso da tecnologia e disponibilizam diversas informação úteis ligadas a produção, contribuem absolutamente para o desenvolvimento da sojicultura, sobretudo por se tratar de uma atividade promissora.

A promoção de ações cooperativas constitui, também, como uma atividade importante no desenvolvimento da sojicultura, porque ao aglutinarem forças e ideias, os produtores terão maior poder de decisão e alcance de oportunidades o que pode traduzir no fortalecimento da atividade e da cadeia global. Os sojicultores auferem grande importância ao papel das ONGs na atividade sojícola.

O terceiro fator, explicou $10,601 \%$ da variabilidade total dos dados e foi identificado como Gestão da Produção por reunir variáveis relacionadas com o desempenho gerencial da atividade sojícola, a evolução da produtividade, do custo de produção e a evolução do faturamento nos últimos 3 anos. Essas variáveis impactam grandemente na busca de ganhos na competividade. Pois, se a produtividade se mantiver baixa na medida em que os custos de produção se tornam 
altos, o país não se tornará competitivo. Portanto, a produtividade é um dos fatores mais importantes e centrais para se obter ganhos em competitividade.

As variáveis que compõe o fator, fazem parte do micronível do modelo da competividade sistêmica, que pressupõe que as melhores práticas organizacionais são fatores determinantes para a competitividade, a gestão efetiva de inovações organizacionais e a gestão tecnológica. Portanto, o uso da tecnologia se torna determinante no alcance de níveis maiores de produtividade e na minimização dos custos de produção, o que poderá proporcionar maior faturamento. Então, uma boa gestão da produção em que obtém-se maiores ganhos de produtividade e minimizam-se os custos, contribuirá para o incremento da competitividade da atividade.

O quarto fator, explicou 9,571\% da variância total e foi denominado Ligação e Importância do Governo e das ONGs, porque reuniu as seguintes variáveis: o nível de ligação com o Governo, a importância atribuída ao papel das ONGs e a importância atribuída ao papel do Governo. Portanto, todas essas variáveis fazem parte do metanível do modelo da competitividade sistêmica, que analisa a integração dos atores da cadeia e que pressupõe que o incremento da competitividade depende da ação dos agentes públicos ou privados.

A ligação do Governo com os sojicultores é imprescindível para o processo de crescimento e desenvolvimento da sojicultura ou mesmo da cadeia toda. Por se tratar do órgão que define e regula as políticas públicas, a integração com o públicoalvo dessas políticas é necessária e também para, através disso, haver uma coordenação entre eles e outros atores da cadeia. Feito isso, há mais chances de se atingir níveis altos de competitividade. As políticas públicas criam condições básicas nacionais e internacionais das atividades locais para garantir estabilidade macroeconômica, incremento da produtividade, inovação e competitividade.

Pelo seu contributo na sociedade, os sojicultores auferem importância ao papel do governo e, além desse órgão, os produtores recebem ajuda em várias áreas de diversas ONGs existentes no país, dando importância também ao papel delas no desenvolvimento das regiões, sobretudo no desenvolvimento agrícola.

O quinto fator, que explicou $9,284 \%$ da variância total, foi denominado Critérios de seleção dos fornecedores de insumo, por reunir variáveis que tem influência na escolha dos fornecedores de insumo: o preço do insumo e a proximidade dos fornecedores. O preço do insumo tem influência no processo de aquisição e uso de tecnologia. Portanto, num período em que se pretende expansão 
e desenvolvimento da cultura e do uso dos pacotes tecnológicos, os preços dos insumos praticados no mercado deverão estar acessíveis aos sojicultores. Quanto mais acessíveis estiverem os preços, os sojicultores estarão motivados a utilizar os pacotes tecnológicos, o que pode melhorar os índices de produtividade, tornando-se incentivo para se manterem na produção e aumentar as áreas de cultivo.

A proximidade dos fornecedores além de diminuir os gastos com transporte, pode ser vantajosa também nos casos em que os fornecedores providenciam acompanhamento técnico, como por exemplo nos padrões de sementeira, aplicação de adubos e pesticidas, amanhos culturais, entre outros. Entretanto, a influência que esses dois critérios de seleção de fornecedores de insumo exercem no processo de expansão e desenvolvimento na sojicultura moçambicana, de maneira, impactarão também nas vantagens competitivas da cultura.

O sexto fator, explicou 8,993\% da variância total e foi designado Importância do Financiamento e do Mercado por reunir as seguintes variáveis: a importância atribuída ao acesso ao crédito e ao escoamento de produto. Ao se falar de escoamento de produto, vários aspectos poderão influenciar. Um deles é a garantia de mercado, que é de extrema importância para o crescimento da sojicultura no país, pois mesmo que haja uma evolução dos índices produtivos, sem ela a produção não desenvolverá. Como demonstrado anteriormente, a soja em Moçambique surgiu para atender a demanda da indústria avícola, que é o maior destino da soja in natura, então, existe mercado para a soja no país.

Outro aspecto a ter em conta é a infraestrutura, pois a existência de estradas em boas condições e transporte são imprescindíveis para que o escoamento de produtos ocorra com sucesso e com custos razoáveis.

Por outro lado, o acesso ao crédito exerce influência para o sucesso de quase todas etapas do processo produtivo quando o produtor não tem condições de arcar com todas as despesas. A aquisição de máquinas e insumos, contratação de mão de obra, escoamento do produto, requererem que o produtor tenha condições pagar. E com a tendência de aumento dos preços dos produtos/insumos, cada vez mais os produtores têm a necessidade de obter apoio financeiro para poder se inserir no mundo da agricultura moderna.

Este nível contempla variáveis com grande influência no crescimento e desenvolvimento da sojicultura no país, consequentemente, no incremento da competitividade da cadeia toda. Portanto, para a consolidação da atividade sojícola é necessário que estas variáveis estejam garantidas. 
O sétimo fator, que explicou 8,931\% da variância total, foi denominado Principais Objetivos dos Sojicultores por reunir aspetos relacionados ao grau de importância de aumentar a área plantada de soja e ao de aumentar a produtividade. As duas variáveis configuram-se nas estratégias de desenvolvimento da produção do micronível do modelo da competitividade sistêmica, auferindo-lhes imensa importância para a consolidação da sojicultura em Moçambique.

Ao constituir objetivo e importância para o sojicultor, aumentar a produtividade e a área plantada de soja, mostra que os produtores estão dispostos a desenvolver e tornar uma atividade viável, o que se torna um passo grande para a busca de ganhos em competitividade. Para tanto, vários aspectos deverão ser garantidos para a solidificação destes objetivos, a maioria deles foram discutidos nos fatores acima.

Portanto, através da análise fatorial foi possível analisar um conjunto de variáveis, agrupadas a partir de dimensões ocultas que não se consegue revelar analisando, por exemplo, duas variáveis. O agrupamento das variáveis permitiu a interpretabilidade das relações existentes entre elas, ou seja, as suas interdependências e a influência no crescimento e desenvolvimento da sojicultura e impacto na competitividade da cadeia produtiva da soja em Moçambique.

\section{Análise de Agrupamentos ou Cluster}

Esta análise foi utilizada para classificar os sojicultores moçambicanos conforme as diversas características/variáveis de ótica da competividade sistêmica. Para tal, foram utilizados os dois métodos básicos de agrupamento: os hierárquicos e os não hierárquicos, de modo a aproveitar os seus benefícios. O hierárquico serviu para agrupar os produtores de acordo com as características, enquanto o método não hierárquico e a análise de variância ANOVA foram utilizados para identificar qual ou quais das variáveis permitem a separação dos clusters, conforme destacam Fávero et al. (2009).

No primeiro método (hierárquico), calculou-se a matriz de similaridade pela distância quadrática euclidiana para 116 produtores utilizando o método Average Linkage (ligação média ou entre grupos), de modo a agrupar os produtores de acordo com a menor distância média.

A regra da parada foi utilizada para determinar o número de agrupamentos. Esta, consiste em observar a maior diferença entre os coeficientes do esquema de aglomeração ou Agglomeration Schedule. Assim sendo, indicou que os 116 produtores poderiam ser agrupados em quatro grupos: o primeiro agrupou 101 
sojicultores, o segundo 12 sojicultores, o terceiro dois e o quarto formado por apenas uma unidade de produção (Figura 2).

Depois de especificado o número de agrupamentos, empregou-se o segundo método (não hierárquico). Em que iniciou-se com a seleção do método a ser utilizado, nesse caso foi o K-médias, que segundo Fávero et al. (2009) produz apenas uma solução para o número de conglomerados predefinidos. Também 0 método minimiza a variância interna dos grupos e maximiza a variância entre os grupos (HAIR, 2009).

Figura 02 - Grupo de sojicultores moçambicanos formados a partir das similaridades competitivas 
JANEQUE, COSTA

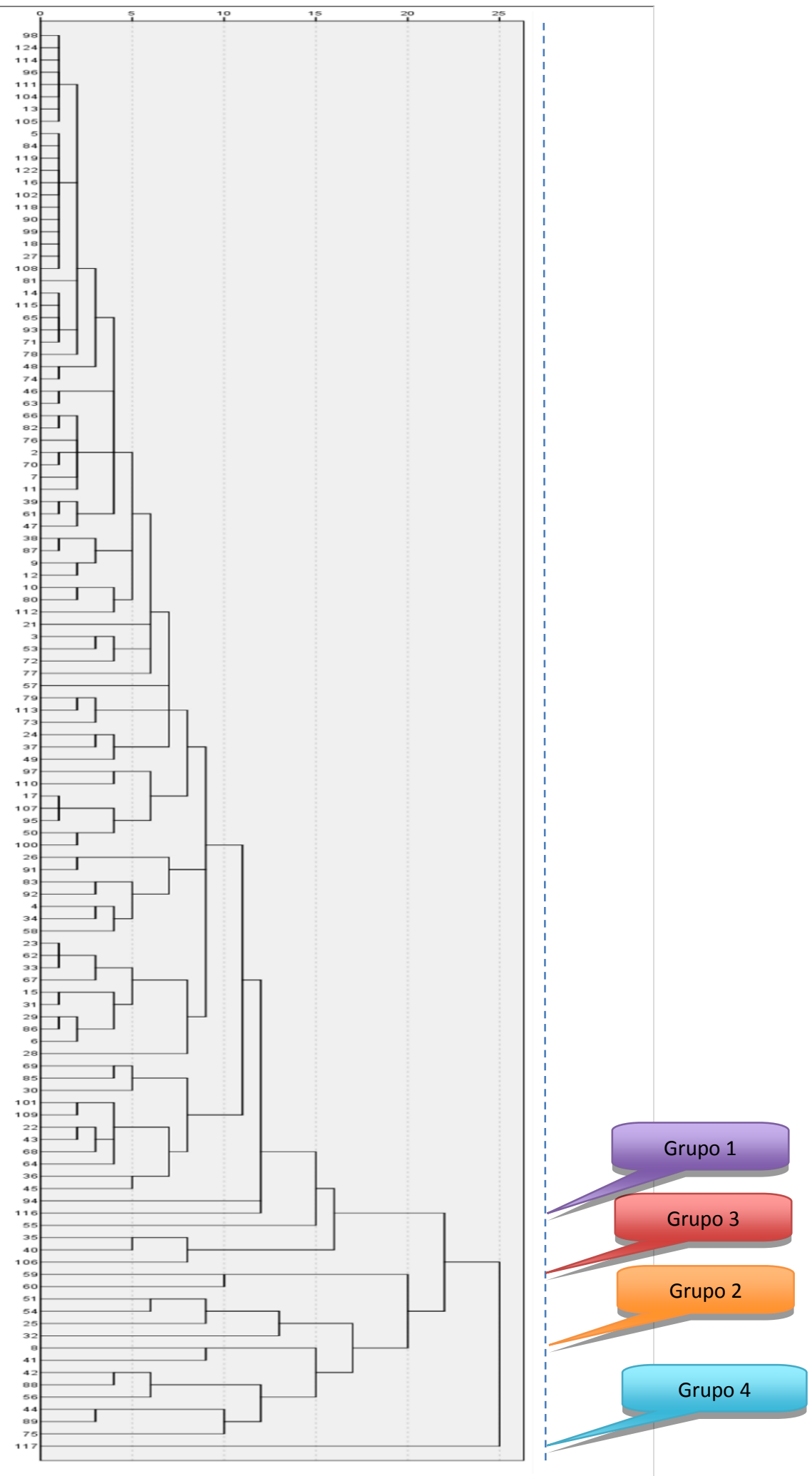

Fonte: Dados da pesquisa de campo (2018)

O procedimento K-médias também é utilizado para verificar se as variáveis utilizadas no procedimento hierárquico são estatisticamente significantes para a formação dos agrupamentos obtidos. Nesse caso, a verificação é feita através da análise de variância (ANOVA) que tem por objetivo identificar qual ou quais das 
variáveis permitem a separação dos clusters, e não verificar se os clusters são ou não diferentes (FÁVERO et al, 2009).

Com isso, foi realizado o teste paramétrico para análise de variância a $5 \%$ de probabilidade, que indicou que 17 variáveis contribuíram mais para a formação dos clusters, o que se pode considerar com 95\% de confiança, que os agrupamentos estão corretamente classificados com relação a essas variáveis. Entretanto, as três variáveis, que não apresentaram diferenças significativas, não possibilitaram distinguir estatisticamente os grupos (Tabela 5).

Pela análise de variância também pode-se constatar que as variáveis V14, V6, V8, V7, V9, V11, V10, V12 e V13, foram as que mais contribuíram para esta formação dos clusters, uma vez que apresentaram as maiores estatísticas $F$.

Tabela 05 - Análise de Variância dos agrupamentos

\begin{tabular}{|c|c|c|c|}
\hline & Descrição da variável & $\mathbf{F}$ & Sig. \\
\hline V1 & $\begin{array}{l}\text {-Vantagem locacional inerente a Proximidade com os fornecedores de } \\
\text { insumos }\end{array}$ & 7,491 & 0 \\
\hline V2 & $\begin{array}{l}\text {-Vantagem locacional inerente a Proximidade com os clientes/ } \\
\text { consumidores }\end{array}$ & 6,193 & 0,001 \\
\hline V3 & $\begin{array}{l}\text {-Vantagem locacional inerente a Infraestrutura física (energia, } \\
\text { transporte, comunicações) }\end{array}$ & 3,877 & 0,011 \\
\hline V4 & $\begin{array}{l}\text {-Vantagem locacional inerente a Disponibilidade de serviços técnicos } \\
\text { especializados }\end{array}$ & 6,162 & 0,001 \\
\hline V5 & $\begin{array}{l}\text {-Vantagem locacional inerente a Existência de programas de apoio e } \\
\text { promoção a produção }\end{array}$ & 6,123 & 0,001 \\
\hline V6 & $\begin{array}{l}\text {-A contribuição de associações, cooperativas locais e ONGs no } \\
\text { tocante a Disponibilização de informações (referentes a matéria-prima, } \\
\text { equipamento, assistência técnica, consultoria, etc.) }\end{array}$ & 81,101 & 0 \\
\hline V7 & $\begin{array}{l}\text {-A contribuição de associações, cooperativas locais e ONGs no } \\
\text { tocante a Promoção de ações cooperativas }\end{array}$ & 50,19 & 0 \\
\hline V8 & $\begin{array}{l}\text {-A contribuição de associações, cooperativas locais e ONGs no } \\
\text { tocante a Promoção de ações dirigidas a capacitação tecnológica de } \\
\text { produtores }\end{array}$ & 69,124 & 0 \\
\hline V9 & -Evolução da produtividade nos últimos 3 anos & 45,255 & 0 \\
\hline V10 & -Evolução do Custo de produção nos últimos 3 anos & 23,545 & 0 \\
\hline V11 & -Evolução do faturamento nos últimos 3 anos & 28,991 & 0 \\
\hline V17 & -A importância atribuída para o Acesso ao crédito & 3,213 & 0,026 \\
\hline V13 & -A importância atribuída para o papel das ONGs & 17,299 & 0 \\
\hline V14 & -A importância atribuída para o papel do Governo & 87,195 & 0 \\
\hline V15 & -A relevância do preço de insumo na seleção de fornecedores & 2,771 & 0,045 \\
\hline V16 & $\begin{array}{l}\text {-A relevância da proximidade dos fornecedores na seleção de } \\
\text { fornecedores }\end{array}$ & 3,014 & 0,033 \\
\hline V18 & -A importância atribuída para o Escoamento de produto & 2,6 & 0,056 \\
\hline V19 & $\begin{array}{l}\text { - O grau de importância de Aumentar a área plantada de soja como } \\
\text { principal objetivo }\end{array}$ & 0,774 & 0,511 \\
\hline V20 & $\begin{array}{l}\text {-O grau de importância de Aumentar a produtividade da soja como } \\
\text { principal objetivo }\end{array}$ & 2,218 & 0,09 \\
\hline V12 & -O nível de ligação com o Governo & 17,332 & 0 \\
\hline
\end{tabular}

Fonte: Dados da pesquisa de Campos (2018)

Com base na dimensão de vantagens locacionais que engloba as variáveis V1, V2, V3, V4 e V5, constata-se que o cluster 4 apresenta melhor vantagem 
locacional e os produtores que compõem o cluster 3 apresentam menor vantagem locacional na maioria dos aspectos, exceto a inerente a infraestrutura física. Entretanto, os produtores dos clusters 1 e 2 apresentam boas vantagens locacionais (Tabela 6). Por outro lado, a dimensão de contribuição associações, cooperativas locais e ONGs que engloba as variáveis V6, V7 e V8, demonstrou que a participação dessas instituições é mais reconhecida por produtores que compõem o cluster 1, ou seja esses produtores receberam mais ajuda por parte das associações, cooperativas locais e ONGs em relação aos sojicultores de outros clusters. 0 cluster 4 recebeu um considerável apoio das instituições em questão. Assim sendo, os produtores dos clusters 2 e 3 representam o grupo de sojicultores isolados.

Tabela 06 - Características dos agrupamentos com base na média das variáveis utilizadas

\begin{tabular}{|c|c|c|c|c|c|}
\hline \multirow{2}{*}{\multicolumn{2}{|c|}{ Descrição das variáveis }} & \multicolumn{4}{|c|}{ Cluster } \\
\hline & & 1 & 2 & 3 & 4 \\
\hline V1 & $\begin{array}{l}\text {-Vantagem locacional inerente a Proximidade com os } \\
\text { fornecedores de insumos }\end{array}$ & 4,673 & 4,333 & 1 & 5 \\
\hline V2 & $\begin{array}{l}\text {-Vantagem locacional inerente a Proximidade com os } \\
\text { clientes/consumidores }\end{array}$ & 4,712 & 4,417 & 1,5 & 5 \\
\hline V3 & $\begin{array}{l}\text {-Vantagem locacional inerente a Infraestrutura física } \\
\text { (energia, transporte, comunicações) }\end{array}$ & 4,772 & 4,5 & 3,5 & 5 \\
\hline V4 & $\begin{array}{l}\text {-Vantagem locacional inerente a Disponibilidade de } \\
\text { serviços técnicos especializados }\end{array}$ & 4,782 & 4,583 & 1,5 & 5 \\
\hline V5 & $\begin{array}{l}\text {-Vantagem locacional inerente a Existência de programas } \\
\text { de apoio e promoção a produção }\end{array}$ & 4,782 & 4,583 & 1,5 & 5 \\
\hline V6 & $\begin{array}{l}\text {-A contribuição de associações, cooperativas locais e } \\
\text { ONGs no tocante a Disponibilização de informações } \\
\text { (referentes a matéria-prima, equipamento, assistência } \\
\text { técnica, consultoria, etc.) }\end{array}$ & 4,663 & 1,083 & 1 & 3 \\
\hline V7 & $\begin{array}{l}\text {-A contribuição de associações, cooperativas locais e } \\
\text { ONGs no tocante a Promoção de ações cooperativas }\end{array}$ & 4,574 & 1,083 & 1 & 3 \\
\hline V8 & $\begin{array}{l}\text {-A contribuição de associações, cooperativas locais e } \\
\text { ONGs no tocante a Promoção de ações dirigidas a } \\
\text { capacitação tecnológica de produtores }\end{array}$ & 4,604 & 1,75 & 1 & 3 \\
\hline V9 & -Evolução da produtividade nos últimos 3 anos & 4 & 3 & 1,5 & 1 \\
\hline V10 & -Evolução do Custo de produção nos últimos 3 anos & 3,584 & 2,583 & 4 & 3 \\
\hline V11 & -Evolução do faturamento nos últimos 3 anos & 3,891 & 3,416 & 2 & 1 \\
\hline V17 & -A importância atribuída para o acesso ao crédito & 4,742 & 4,333 & 4 & 5 \\
\hline V13 & -A importância atribuída para o papel das ONGs & 4,336 & 2 & 3 & 1 \\
\hline V14 & -A importância atribuída para o papel do Governo & 4,178 & 2,5 & 3 & 1 \\
\hline V15 & $\begin{array}{l}\text {-A relevância do preço de insumo na seleção de } \\
\text { fornecedores }\end{array}$ & 3,079 & 2,666 & 3 & 5 \\
\hline V16 & $\begin{array}{l}\text {-A relevância da proximidade dos fornecedores na } \\
\text { seleção de fornecedores }\end{array}$ & 3,118 & 2,666 & 3 & 1 \\
\hline V18 & -A importância atribuída para o Escoamento de produto & 4,841 & 4,583 & 4,5 & 5 \\
\hline V19 & $\begin{array}{l}\text {-O grau de importância de Aumentar a área plantada de } \\
\text { soja como principal objetivo }\end{array}$ & 4,940 & 4,916 & 5 & 1 \\
\hline V20 & $\begin{array}{l}\text {-O grau de importância de Aumentar a produtividade da } \\
\text { soja como principal objetivo }\end{array}$ & 4,990 & 5 & 5 & 1 \\
\hline V12 & -O nível de ligação com o Governo & 3,920 & 3,083 & 1 & 1 \\
\hline
\end{tabular}

Fonte: Dados da pesquisa de campo (2018) 
O cluster 1 apresentou melhores indicadores microeconômicos que englobam as variáveis V9, V10 e V11, e os clusters 3 e 4 os piores indicadores. Entretanto, os produtores do cluster 3 apresentaram maior evolução do custo de produção nos últimos três anos (V10) em relação aos produtores dos demais clusters. Portanto, os sojicultores que receberam mais apoio das associações, cooperativas locais e ONGs (cluster 1) foram os que tiveram melhor desempenho produtivo e os que tiveram menos contribuições demonstraram ser menos competitivos.

Os sojicultores do cluster 1 foram os que mais reconheceram e auferiram muita importância ao papel do governo e das ONGs (V13, V14), provavelmente por, ao nível dos clusters, serem os que apresentam uma considerável ligação com estas entidades. O cluster 4, demostrou ter baixíssima ou inexistente ligação com 0 governo (V12) e auferiu nenhuma importância ao papel desta entidade e das ONGs. Por outro lado, os produtores do cluster 3 apesar de apresentar baixíssima ou inexistente ligação com o governo, atribuem razoável importância ao papel das entidades acima. Já os sojicultores do cluster 2 tiveram uma razoável ligação com o governo, mas concedem menos importância ao papel do governo e das ONGs.

Portanto, os sojicultores do cluster 1, provavelmente apresentaram maior evolução da produtividade nos últimos 3 anos (V9) por terem maior ligação com o governo e terem tido maior contribuição das associações, cooperativas locais e ONGs.

Na dimensão de critérios de seleção dos fornecedores de insumo, o cluster 4 concedeu mais relevância ao preço de insumo e irrelevância a proximidade com fornecedores de insumos. Os sojicultores dos clusters 1 e 3 atribuíram razoável importância a esses critérios de escolha dos fornecedores, por conseguinte os critérios representam pouca relevância para os sojicultores do cluster 2.

Quanto a importância concedida ao acesso ao financiamento e ao escoamento da produção, para o cluster 4 essas variáveis constituem extrema importância para a sojicultura enquanto que os sojicultores dos demais agrupamentos atribuíram muita importância para estes itens.

Com relação aos principais objetivos, os sojicultores dos clusters 1,2 e 3 atribuíram extrema importância aumentar a área plantada e a produtividade da soja, por conseguinte isso não constitui importância para cluster 4.

Com esses resultados é possível afirmar que os sojicultores do cluster 1 são os que estão mais engajados na evolução da sojicultura moçambicana e recebem mais contribuição de políticas públicas e privadas. Os do cluster 2 podem ser 
considerados aqueles com média evolução produtiva e com menos apoio institucional. Já os sojicultores dos clusters 3 e 4 representam os que tiveram menos evolução da produtividade, mas diferem na contribuição institucional obtida. Sendo que o cluster 4 o que recebeu mais apoio e o 3 nenhum apoio.

Portanto, com a análise de agrupamento foi possível agrupar os sojicultores das principais regiões produtoras de Moçambique em quatro clusters conforme as características inerentes a competitividade sistêmica. Esse agrupamento ajudou a estudar as características comuns dos produtores de cada grupo e assim caracterizar o tipo de sojicultores entrevistados. A outra técnica multivariada exploratória (análise fatorial) também auxiliou a formar dimensões (fatores) que sintetizaram determinadas características/variáveis dos níveis analíticos da competitividade sistêmica.

\section{Considerações Finais}

A sojicultura está cada vez mais se tornando uma atividade importante para vários países, especialmente para Moçambique. Em que, verifica-se uma transformação socioeconômica das zonas rurais, notadamente na utilização de tecnologias agrícolas; participação da soja na dieta alimentar, o que indiretamente combate os problemas de desnutrição da população; diversificação da renda dos produtores, pois é considerada cultura de rendimento; entre outras.

Os resultados deste estudo, permitiram interpretar as dinâmicas de competitividade dos sojicultores das principais regiões produtoras de Moçambique. Demonstrando a importância das políticas públicas e privadas para o desenvolvimento do cultivo de soja no país, pois verificou-se que os produtores que recebem mais assistência técnica apresentaram melhores desempenhos produtivos em relação aos outros que recebem menos ou nenhuma assistência. Isso corrobora com o modelo da competitividade sistêmica, base deste estudo, que pressupõe que a integração dos atores das cadeias produtivas, a contribuição de instituições e ação dos agentes públicos ou privados auxiliam no incremento da competitividade.

Além disso, a pesquisa demonstrou ainda a importância da existência de boas condições de infraestrutura e a disponibilização de financiamento/crédito para evolução e consolidação da sojicultura no país. Isso, mostra outra vez a necessidade de uma política pública que enaltecerá as condições atuais para melhores, sobretudo no uso de tecnologias. Entretanto, esses fatores influenciarão 
na gestão da produção na unidade de produção, sobretudo em ganhos de produtividade, aumento do lucro e controle de custos - principais condicionantes de competitividade.

Contudo, apesar da pesquisa basear-se em técnicas com um nível significativo de robustez, os resultados adquiridos podem aliciar novos estudos, ou seja, deixa-se aberto para outras contribuições dos mais diversos pesquisadores. Portanto, foi possível concluir que a ação de políticas públicas e privadas nas regiões em estudo, proporcionaram melhores índices produtivos da sojicultura, incitando a sugestão de incremento e melhoria das ações dessas políticas, sobretudo no tocante na assistência técnica e na disponibilização de tecnologias, ingredientes importantes para a obtenção de ganhos em competitividade.

\section{REFERÊNCIAS}

CAP - CENSO AGRO-PECUÁRIO 2009 - 2010: RESULTADOS DEFINITIVOS MOÇAMBIQUE. Ficha Técnica. Editor: Instituto Nacional de Estatística - Moçambique. 2011.

CORRAR, Luiz J.; PAULO, Edilson; DIAS FILHO, José Maria. Análise multivariada: para os cursos de administração, ciências contábeis e economia. Atlas, São Paulo, 2017.

COSTA, N. L.; SANTANA, A. C.; BASTOS, A. P. V.; BRUM, A. L. Desenvolvimento tecnológico, produtividade do trabalho e expansão da cadeia produtiva da soja na Amazônia Legal. In: SANTANA, A. C. (Org.). Mercado, cadeias produtivas e desenvolvimento rural na Amazônia. 1ed.Belém/PA: UFRA, 2014, v. 1, p. 81 -112.

CostA, N., COSTA, V., Mattos, C. A., TEIXEIRA, O., FLORES, A., \& OLIVEIRA, G. (2017). Capital Humano e Desenvolvimento Econômico no Rio Grande do Sul: Uma Abordagem Multivariada. Desenvolvimento em Questão, 15(38), 380-402.

https://doi.org/10.21527/2237-6453.2017.38.380-402

EMPRESA BRASILEIRA DE PESQUISA AGROPECUÁRIA - EMBRAPA. O agronegócio da soja nos contextos mundial e brasileiro. 2014.

ESSER, K. et al. Competitividad sistémica: nuevo desafio a las empresas y a la política. Revista da CEPAL, Santiago, n. 59, 1996.

ESSER, K.; HILLEBRAND, W.; DIRK, M.; MEYER STAMER, J. Competitividad sistémica: competitividad internacional de las empresas y políticas requeridas. Berlín: Instituto Alemán de Desarrollo, 1994.

FÁVERO, L. P. et al. Análise de dados: modelagem multivariada para tomada de decisões. São Paulo: Campus, 2009. 
GIL, A. C. 2002. Como elaborar projetos de pesquisa. 4. ed. São Paulo: Atlas, 2002.

HAIR JR., J. F. et al. Análise multivariada de dados. Porto Alegre: Bookman, 2009.

HAIR, Jr; BLACK, W. C; BABIN, B. J; ANDERSON, R. E e TATHAM, R. L. Multivariate Data Analysis. 6ª edição. Upper Saddle River, NJ: Pearson Prentice Hall, 2006.

JOHNSON, R. A.; WICHERN, D. W. Applied multivariate statistical analysis. New York: Prentica-Hall, 1992.

MINGOTI, S. A. Análise de dados através de métodos de estatística multivariada, uma abordagem aplicada. 1를 Belo Horizonte, Editora UFMG, 2007.

PEREIRA, L.; YAN, I. 2012. Cadeia de Valor soja, Boletim Informativo soja, Ed. № 9. 2012.

REIS, E.A., REIS, I.A. Análise Descritiva de Dados. Relatório Técnico do Departamento de Estatística da UFMG. 2002. Disponível em: www.est.ufmg.br

RITA, Santa L. P.; JúNIOR, Fereira R.R. Agências de Fomento: Um Mecanismo Indutor de Arranjos Produtivos Locais para o Desenvolvimento Regional do Estado de Alagoas. Anais do XI Seminario Latino-Iberoamericano de Gestión Tecnológica - ALTEC. Salvador, 2005

SANTANA, A. C. A competitividade sistêmica das empresas de madeira da Região Norte. FCAP, Belém, 2002.

SMART, T; HANLON, J. Chickens and beer: A recipe for agricultural growth in Mozambique. United Kingdom: The Open University. 2014.

SUDENE. Bases para a recriação da SUDENE por uma política de desenvolvimento sustentável para o Nordeste. Pernambuco: SUDENE, 2003.

TECHNOSERVE BUSINESS SOLUTION TO POVERTY. Southern Africa regional soybean roadmap. Washington, DC: TechnoServe, Feb. 2011. 57 p.

WALKER, T.; CUNGUARA, B. Avaliação da Situação da P\&D da Soja e o Programa "Feed the Future", da USAID, em Moçambique em 2016. Relatório de Pesquisa 81P, Ministério da Agricultura e Segurança Alimentar, Direcção de Planificação e Cooperação Internacional: Maputo. 2016.

\section{NOTAS DE AUTOR}

\section{CONTRIBUIÇÃO DE AUTORIA}

Ricardina António Janeque - Concepção e elaboração do manuscrito. Coleta de dados, Análise de dados, Elaboração do manuscrito, revisão e aprovação da versão final do trabalho

Nilson Luiz Costa - Concepção e elaboração do manuscrito. Análise de dados, Elaboração do manuscrito, revisão e aprovação da versão final do trabalho.

\section{FINANCIAMENTO}

O presente artigo baseia-se na dissertação de Mestrado em Agronegócios da primeira autora, junto à Universidade Federal de Santa Maria (UFSM) e foi apoiado pela Agência dos Estados Unidos para o

Desenvolvimento Internacional, como parte da iniciativa Feed the Future, no âmbito do Fundo CGIAR, número de 
prêmio BFS-G-11-00002, e o antecessor financia a Segurança Alimentar e a concessão da Mitigação da II Crise, número de prêmio EEM-G-00-04-00013. O estudo também contou com apoio logístico da Universidade Zambeze (Moçambique), sendo este fundamental para a realização da coleta de dados primários. Também, a pesquisa recebeu apoio da Coordenação de Aperfeiçoamento de Pessoal de Nível Superior - CAPES (Brasil) - Código de Financiamento 001.

\section{CONSENTIMENTO DE USO DE IMAGEM}

Não se aplica.

\section{APROVAÇÃO DE COMITÊ DE ÉTICA EM PESQUISA}

De acordo com o Parágrafo único do Art. 1ํ da Resolução № 510, de 07 de abril de 2016, do Conselho Nacional de Saúde, não se aplica.

\section{CONFLITO DE INTERESSES}

Não se aplica.

\section{LICENÇA DE USO}

Este artigo está licenciado sob a Licença Creative Commons CC-BY. Com essa licença você pode compartilhar, adaptar, criar para qualquer fim, desde que atribua a autoria da obra.

\section{HISTÓRICO}

Recebido em: 07-05-2020

Aprovado em: 01-02-2021 